\title{
Evidence for familial aggregation in siblings with autoimmune rheumatic diseases
}

\author{
Madalina-Pusa Duna', Denisa Predeteanu' ${ }^{1}$, Florian Berghea ${ }^{1,2}$, Mihai Abobului ${ }^{1,2}$, \\ Violeta Vlad', Andra Balanescu ${ }^{1,2}$, Daniela Opris-Belinski ${ }^{1,2}$, Violeta Bojinca ${ }^{1,2}$, \\ Cosmin Constantinescu ${ }^{1,2}$, Andreea Borangi ${ }^{1,2}$, Laura Groseanu ${ }^{1,2}$, Ioana Saulescu ${ }^{1,2}$, \\ Maria-Magdalena Negru',2, Claudia Cobilinschi',2, Sanziana Daia ${ }^{1,2}$, \\ Diana Mazilu ${ }^{1,2}$, Ruxandra Ionescu, ${ }^{1,2}$ \\ ${ }^{1}$ Department of Internal Medicine and Rheumatology, "Sf. Maria" Clinical Hospital, Bucharest, Romania \\ 2"Carol Davila" University of Medicine and Pharmacy, Bucharest, Romania
}

\begin{abstract}
Autoimmune rheumatic disorders have a multifactorial determinism, caused by various environmental factors acting on the individual's genetic susceptibility, destabilizing the systems which regulate the immune response. Epidemiological and genetic investigations are very important to demonstrate the contribution of genetic factors to the development of these autoimmune diseases. The contribution of genetic factors in causing autoimmune diseases has been demonstrated by familial aggregation. Moreover, it was also quantified by determining heritability, expressing the proportion of genetic factors in the etiology. It is now clear that common genes underlie multiple autoimmune disorders.
\end{abstract}

Keywords: familial aggregation, siblings, autoimmune disorders

\section{INTRODUCTION}

Autoimmune diseases (ADs) share a number of characteristics that suggest common etiologic pathways or mechanisms, including reactivity to selfantigens by the humoral and/or cellular immune system, as well as genetic associations with human leukocyte antigen (HLA). Although familial clustering of autoimmunity has long been recognized, the patterns of aggregation across different autoimmune diseases have not been clearly delineated (1). Among the more common autoimmune disorders, probably the best evidence for familial clustering among different autoimmune rheumatic diseases involves rheumatoid arthritis (RA), ankylosing spondylitis (AS), systemic lupus erythematosus (SLE) and others rheumatic diseases (2).

\section{OBJECTIVE}

The objectives of the study is to determine whether there is familial aggregation of autoimmune diseases in patients known with rheumatoid arthritis, ankylosing spondylitis, systemic lupus erythematosus and others autoimmune rheumatic diseases. The aim of the study is to evaluate the aspects of familial aggregation of autoimmune rheumatic diseases in a group of patients diagnosed with rheumatoid arthritis, ankylosing spondylitis, systemic lupus erythematosus, systemic sclerosis, polymyositis, psoriatic arthritis and mixed connective tissue disease.

\section{METHOD}

We realized a cross-sectional study by enrolling 90 patients diagnosed with rheumatoid arthritis, ankylosing spondylitis, systemic lupus erythematosus, systemic sclerosis, polymyositis, psoriatic arthritis and mixed connective tissue disease. I have selected only those patients who have relatives with autoimmune rheumatic diseases. The group included patients admitted between 1 January 2015 and 31 December 2017 in the clinic of Internal Medicine and Rheumatology of the Saint Mary Hospital Bucharest. The data was extracted from each patient's clinical files. For each patient we followed: demograph- 
ic variables, sex, the year of the disease's onset, disease-related and therapeutic-related parameters, the degree of kinship and familial aggregation. We included in the study also family members of patients with autoimmune diseases. We used questionnaires to obtain data on family and personal medical history and an informed consent was signed for each patient.

\section{RESULTS}

The study included 90 patients with autoimmune rheumatic diseases 51 females (56.66\%) and 39 males $(43.34 \%)$, with the mean age $50-69$ years.
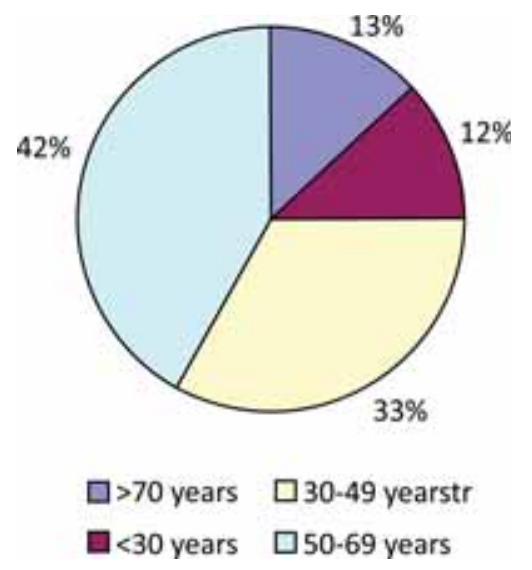

FIGURE 1. Agewise distribution of cases $(n=90)$

First-degree relatives were most affected $(n=49)$. Among the patients diagnosed with RA (32 patients), 26 of them $(81.25 \%)$ have relatives with RA, and among those, 15 are first-degree relatives. 4 rheumatoid patients have their relatives diagnosed with LES (sister, mother) and 2 patients have their father and mother diagnosed with systemic sclerosis.

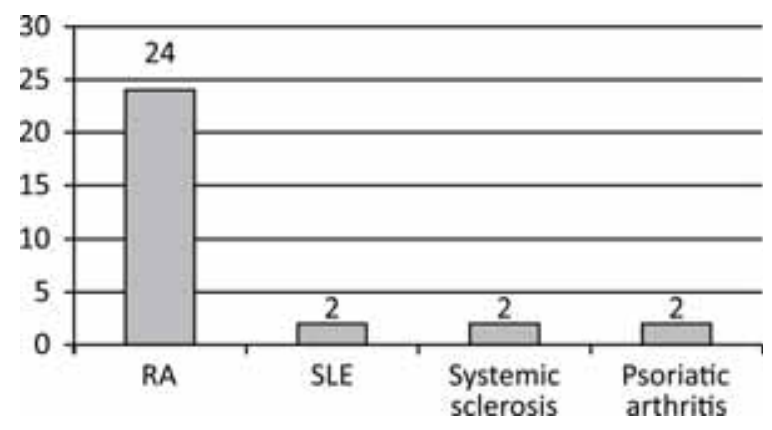

FIGURE 2. Family history of RA patients

Among the 24 ankylosing spondylitis patients, it seems that 19 of them have relatives with the same condition, 11 of them being first-degree relatives. The other patients have their close relatives known with rheumatoid arthritis (3 relatives), psoriatic arthropathy (one relative) and Crohn's disease (one relative) which is a diseases captured under the umbrella of spondyloarthritis.

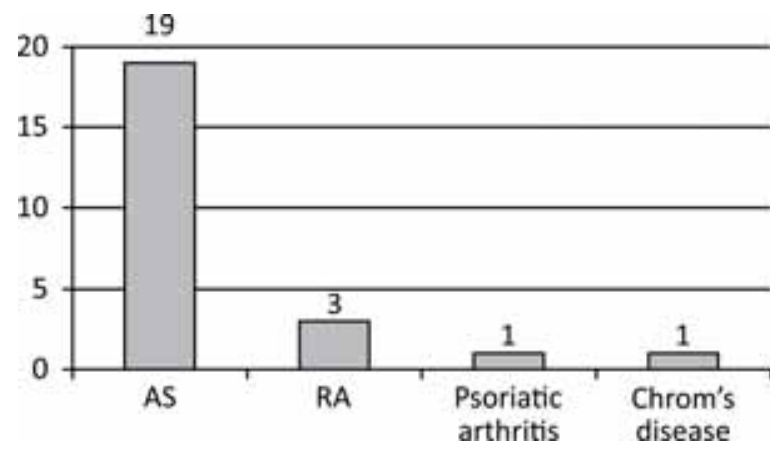

FIGURE 3. Family history of AS patients

Regarding the patients with SLE, almost half of them had relatives with SLE, but no first degree relative, aspect discordant with literature data which says that higher frequency occurs in first degree relatives and $5 \%$ of the relatives have the disease, and $10 \%$ have another autoimmune disease (3). 4 SLE patients have their mothers diagnosed with rheumatoid arthritis. $84.6 \%$ of lupus patients were treated with hydroxychloroquine for lupus skin involvement, musculoskeletal and hematological manifestation. 2 of the patients were treated with mycophenolate mofetil for lupus nephritis. All the patients have the immunologic criteria, those required in SLICC classification system.

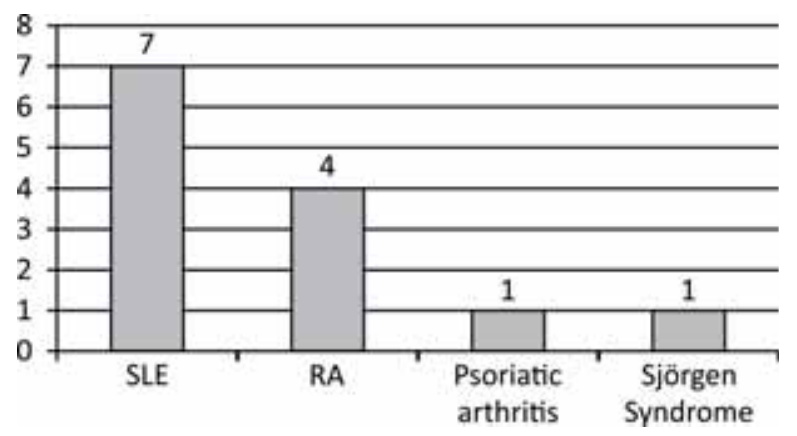

FIGURE 4. Family history of SLE patients

Four articles assessed familial autoimmunity in SLE probands. Alarcon-Segovia et al. evaluated familial aggregation in the, Grupo Latinoamericano de Estudio de Lupus' (GLADEL). They found that among all family members who had any autoimmune diseases, 6.7\% had RA and other ADs at a lesser frequency (4). In first degree relative (FDR) $(\mathrm{n}=114)$ with ADs, $28 \%(\mathrm{n}=32)$ had RA. Likewise, an increased frequency of familial autoimmunity 
was found in SLE probands compared with population prevalence. Priori et al. found an odds ratio of 4.6 (95\% confidence interval 1.94 to 11.1$)$ in a multivariate analysis of familial autoimmunity in FDR of SLE patients. They reported autoimmune thyroid disease (AITD) as the most frequent disease with eight cases, followed by RA with five cases, vitiligo with three cases and type 1 diabetes with two cases. Psoriasis frequency was higher among non-autoimmune controls. Sjögren's syndrome (SS) as well as AITD were described by Scofield et al. while Corporaal et al. found clustering of RA.

The number of patients with psoriatic arthritis prevailed relatives with psoriasis vulgaris in $75 \%$ of cases and not psoriatic arthritis, which could be interpreted as a mistake in diagnosis of these patients. Only one patient had his father with psoriatic arthritis, both treated with methotrexate.

Patients with systemic sclerosis did not have relatives with the same condition in the family. The firstdegree relatives of systemic sclerosis patients were diagnosed with rheumatoid arthritis and with SLE. As far as we know, familial aggregation of systemic sclerosis and co-aggregation with other autoimmune diseases is rarely reported ${ }^{5}$. However, the risks of systemic sclerosis and other autoimmune diseases are increased in relatives of people with systemic sclerosis, and family factors explain over two-thirds of the phenotypic variance of the disease. These findings may be useful in counselling families of patients with systemic sclerosis and for further genetic studies (6).

We identified 3 parent-child relationships regarding the mixed connective tissue disease. The daughter of a patient had prominent SLE features and kidney involvement, and the mothers of the other patients had rheumatoid arthritis and Sjögren syndrome. All the patients with definite diagnosis of mixed connective tissue disease had positive antiribonucleoprotein antibodies.

\section{DISCUSSION}

The study of genes involved in the immune response in antigen presentation in the formation of autoantibodies, is directions to follow in the future. Genetic studies will find their usefulness in drawing up family surveys and population studies on rheumatic autoimmune diseases (7). The coexistence in the same family with several different autoimmune diseases supports the hypothesis that certain genes can induce autoimmune disease predisposition to many diseases.

In this project we attempt to give a broad overview of the fast moving field of human genetics as it applies to the problem of human autoimmunity by studying haplotypes for each disease in order to see if there is familial aggregation and if there is any connection between genes and response to treatment . Maybe will know if that certain genes may explain the pathogenic mechanism and can influence the disease phenotype. An important thing is to perform screening tests in patients with family history of autoimmune disease for a more accurate and early diagnosis which cand lead us to an earlier treatment, prevent and delay complications caused by the disease, improved quality of life.

In our study first-degree relatives were most affected $(n=49)$, rheumatoid arthritis and ankylosing spondylitis followed by SLE were the most frequent autoimmune diseases encountered. Among the patients diagnosed with RA (32 patients), 26 of them (81.25\%) have relatives with RA, among the 24 ankylosing spondylitis patients, it seems that 19 of them have relatives with the same condition, 11 of them being first-degree relatives.

Regarding the patients with SLE, almost half of them had relatives with SLE, but no first degree relative, aspect discordant with literature data which says that higher frequency occurs in first degree relatives.

We identified 8 patients with psoriatic arthritis, first and second degree relatives and 6 of them had relatives with psoriasis vulgaris expressed by the presence of facial, hand and foot psoriasis. Only one psoriatic arthritis patient had his father with psoriatic arthritis. One interesting thing that I noticed was that higher socioeconomic level were more likely to have familial autoimmunity.

\section{CONCLUSIONS}

The results found in this work support aggregation of diverse autoimmune diseases (ADs) (that is, familial autoimmunity) and the view of a common origin for ADs. Familial autoimmunity is still a topic that has not been thoroughly explored.

The coexistence of several different autoimmune diseases in the same family supports the hypothesis that certain genes can increase the predisposition to many diseases (8). 
The genetic studies will predict the risk of developing autoimmune diseases with family aggregation in the future.

Familial autoimmunity is a frequently seen condition. Further study of familial autoimmunity will help to decipher the common mechanisms of autoimmunity.

\section{REFERENCES}

1. Heward J. Gough S.C.L. Genetic susceptibility to the development of autoimmune disease, 1997, 93: 479-491.

2. Davidson A., Diamond B. Autoimmune diseases, 2001: 340-350.

3. Dausset J., and Svejgaard A. (1977). HLA and Disease. Munksgaard and Williams and Wilkins, Copenhagen and Baltimore.

4. Jorge Cardenas-Roldan et al. How do autoimmune diseases cluster in families? A systematic review and meta-analysis, BMC Medicine, Published online 2013 Mar 18. doi: 10.1186/1741-701511-73.

5. Vanesa Pytel, Familial multiple sclerosis and association with other autoimmune diseases, Brain and Behavior, Volume 8, Issue 1
The clinical and etiological heterogeneity of autoimmune diseases and understanding the relationship between genotype and phenotype are the important steps in genetic mapping.

Conflict of interest: none declared Financial support: none declared

6. Kuo C.F. et al. Familial risk of systemic sclerosis and coaggregation of autoimmune diseases in affected families, Arthritis Res Ther. 2016 Oct 12;18(1):231.

7. Lindsey A. Criswell et al. Analysis of Families in the Multiple Autoimmune Disease Genetics Consortium (MADGC) Collection: the PTPN22 620W Allele Associates with Multiple Autoimmune Phenotypes, Am J Hum Genet. 2005 Apr; 76(4): 561-571. Published online 2005 Feb 17. doi: 10.1086/429096

8. Bluestone R. (1977). In Immunogenetics and Rheumatic Disease, edited by D.A. Brewerton. Saunders, London. 